\title{
DEPENDENCE OF ELECTROSTATIC FIELD ON PROPERTIES OF PAPER IN A TWO-DIMENSIONAL TONER IMAGE TRANSFER NIP
}

\author{
J. Kaladė ${ }^{\mathrm{a}}$, R. Maldžius ${ }^{\mathrm{a}}$, J. Sidaravičius ${ }^{\mathrm{b}}$, P. Sirviö ${ }^{\mathrm{c}, \mathrm{d}}$, and K. Backfolk ${ }^{\mathrm{c}}$ \\ ${ }^{a}$ Vilnius University, Saulètekio 9, LT-10222 Vilnius, Lithuania \\ ${ }^{\mathrm{b}}$ Vilnius Gediminas Technical University, J. Basanavičiaus 28, LT-03224, Lithuania \\ E-mail: jonas.sidaravicius@takas.lt \\ ${ }^{\mathrm{c}}$ Stora Enso Oyj, Imatra Research Centre, FI-55 800 Imatra, Finland \\ d Åbo Akademi University, Department of Physical Chemistry, Porthaninkatu 3-5, FI-20 500 Turku, Finland
}

Received 3 December 2008; revised 1 May 2009; accepted 18 June 2009

\begin{abstract}
Electrostatic fields in the toner image transfer nip of laser printers were calculated by exact solution of the Poisson equations for a two-dimensional (2D) model and by applying for the paper the approximation of dielectric thickness. Both the distribution and location of the electric field strength component parallel to the paper surface were investigated and field values were calculated for different paper and toner parameters. The conditions of the occurrence of Paschen discharge in the toner image transfer nip were analysed.
\end{abstract}

Keywords: electrostatic fields, Poisson equation, electrophotography, toner transfer, paper

PACS: $41.20 . \mathrm{Cv}, 42.30 . \mathrm{Va}$

\section{Introduction}

In electrophotographic printers and copiers, the toner image is transferred from the photoreceptor onto the paper or onto an intermediate belt using an electric field. The quality of the transferred image depends on the electric field strength and its configuration in the transfer nip. Various aspects of toner image transfer have been widely investigated [1-11]. An image transfer system is frequently quite complicated with a complex symmetry (Fig. 1), the paper is dielectrically inhomogeneous, and an exact mathematical description of charge transfer (i. e., of transfer of the charged toner particles) is therefore especially complex. For this reason the image transfer dynamics are usually considered in terms of a simplified one-dimensional (1D) model $[1,4,7]$ or a model based on the assumption that the field lines are perpendicular to every surface of the system layer $[2,6]$. The latter assumption allows one to calculate the vertical component of the electric field perpendicular to the paper surface in a complex system though there is no exact justification for such approximation. Besides it is then impossible to evaluate the horizontal electric field component parallel to the paper surface, which is important in practice. The parallel component caused by the paper roughness has been evaluated using the

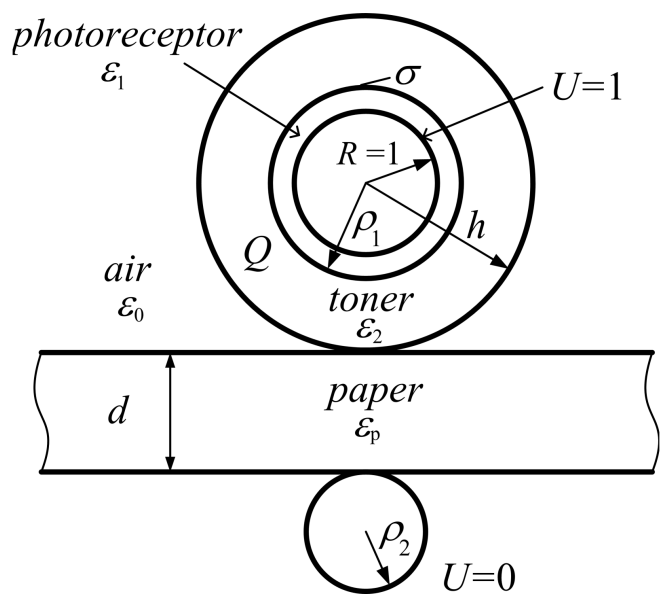

Fig. 1. Diagram of the system 'photoreceptor cylinder - toner (PCT) - paper - transfer cylinder', where $\sigma$ is the surface charge density at the semiconductor-toner interface, $Q$ is the toner space charge density, $\rho_{1}$ is the semiconductor cylinder radius, $h$ is the distance from the PCT center to the toner edge, $d$ is the paper thickness, $\rho_{2}$ is the radius of the transfer cylinder (all quantities are dimensionless; see the text).

method of finite elements [8] and it has been found to be quite small - about $1 \%$ of the perpendicular electrical field value. In addition, it has been shown [8] that values of the perpendicular component obtained using the 1D model may differ from the exact values obtained numerically by a factor greater than 2 . It is clear that these 
models are only a crude approximation of a real image transfer system. Not only the mentioned paper dielectric inhomogenity and surface roughness, but also the geometry of a real system can have an essential role in the distribution of electrostatic field. In the toner-paper interface region the electric field has not only a vertical component perpendicular to the paper surface but also a horizontal component parallel to the paper surface. The latter component, together with the Paschen effect, can play role in the widening of the transferred image and its quality. The role of the geometry on the electric field distribution in the toner transfer nip is not investigated in detail but the rising requirements to the image quality make important the investigation of the impact of the geometry of toner transfer nip.

Various researches $[8,10,11]$ have investigated the influence of the dielectric heterogeneity of paper and the role of paper microstructure on the toner image transfer using modified 1D models. Finite element methods can be used to study variation in the electric field $z$ component with local variations in paper structure and dielectric properties, although the horizontal behaviour remains unclear.

A similar problem of calculating the electric field strength in a system with a complex configuration arises in the analysis of the photoreceptor charging by the cylinder [12]. In this case, electric fields have been evaluated using a 1D model and it is claimed that the results are in good agreement with experimental data, although there is no analysis of the limits of applicability of the 1D approximation [12].

The present work is not concerned with problems of charge dynamics and paper inhomogeneity. Instead, the aim is to obtain exact solutions of the Poisson equation taking into account the 2D geometry of the system, in order to determine properties of electrostatic field in a toner image transfer system and to evaluate the parallel to the paper surface electric field component. Expressions of the photoreceptor and toner electrostatic potential are derived, the case of infinite dielectric permittivity of paper is analysed, and the highest value of electrostatic field strength is determined. Also the common methods for the calculation are formulated and other cases of paper parameters are analysed using the approximation of dielectric thickness.

Exact calculation of the electric field distribution and its dependence on the transfer nip geometry and paper dielectric permittivity and thickness enables one to predict the conditions of the occurrence of Paschen discharge. The results obtained may be important not only for the definition of initial conditions for the transfer of

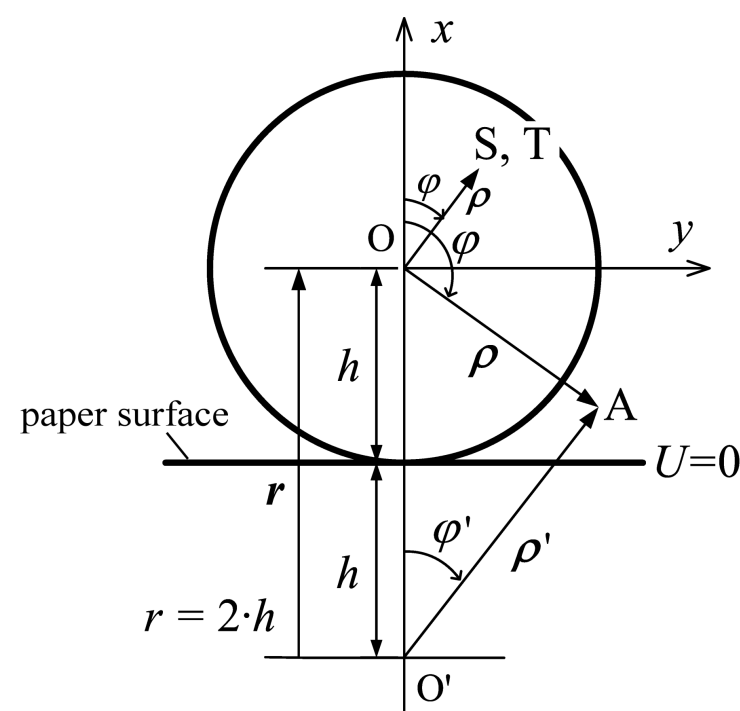

(a)

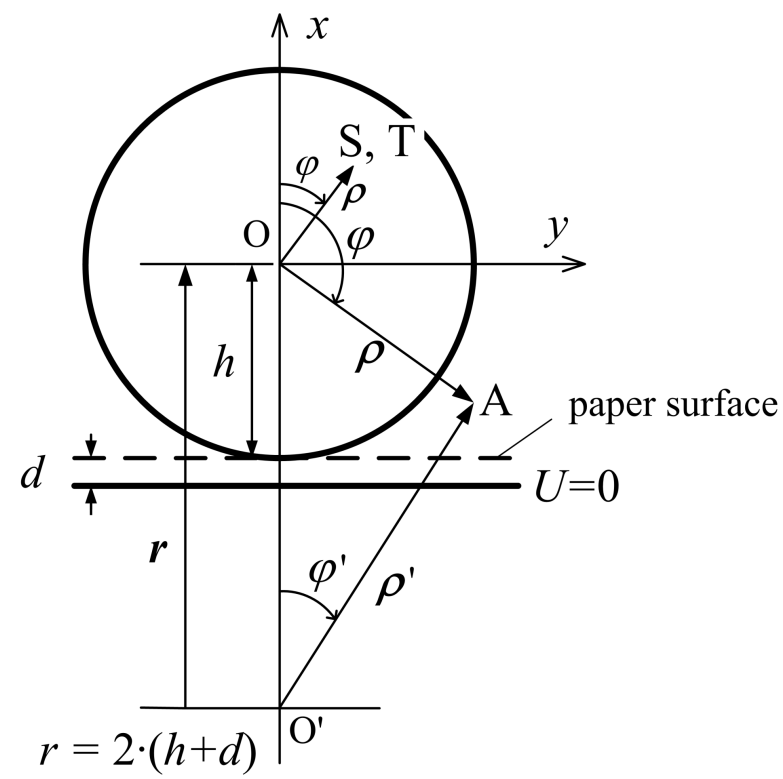

(b)

Fig. 2. Diagrams of the investigated system in polar coordinates: (a) $\varepsilon_{\mathrm{p}}=\infty$ and (b) $\varepsilon_{\mathrm{p}}=\varepsilon_{0}$. S, T are the points in the semiconductor and in the toner respectively; $\mathrm{A}$ is a point in the air.

charged toner particles, but also for the evaluation of results of 1D models.

\section{Distribution of electrostatic potential in the cylinder-semiconductor-toner system}

In all cases considered in this work, the photoreceptor cylinder of organic semiconductor was covered by a constant thickness toner layer (PCT) and only the photoreceptor and the toner properties (thickness, dielectric permittivity) were varied. The system is unlimited in the $z$ direction perpendicular to the plane of the cross- 
section shown in Fig. 1, and it is symmetric relative to the $x O z$ plane (Fig. 2).

The radius of the metal cylinder coated with the semiconductor layer is denoted $R(\mathrm{~cm})$, and its potential, which is equivalent to the toner image transfer potential, is denoted $U_{0}(\mathrm{~V})$. The potential of the transfer cylinder is zero. In the analysis, if not stated otherwise we use dimensionless quantities: distances being expressed in units of $R$, and potentials in units of $U_{0}$. Using such units, the radius of the cylinder and its potential are equal to unity (Fig. 1), the paper thickness $d$ is equal to the actual paper thickness $d_{\mathrm{dim}}(\mathrm{cm})$ divided by $R(\mathrm{~cm})$, i. e., $d=d_{\mathrm{dim}} / R$, all dielectric permittivities are relative to the air dielectric permittivity. The latter value is $\varepsilon_{0}=1$ but in the text it is denoted as $\varepsilon_{0}$. Dimensionless electric field strength is obtained after dividing the dimensional electric field strength by cylinder radius: $U_{0}(\mathrm{~V}) / R(\mathrm{~cm})$ etc. The distribution of the potential $U$ in the PCT part is then described in polar coordinates $\rho$ and $\varphi$ (Fig. 2) by the following equation:

$$
\frac{\partial^{2} U}{\partial \rho^{2}}+\frac{1}{\rho} \frac{\partial U}{\partial \rho}+\frac{1}{\rho^{2}} \frac{\partial^{2} U}{\partial \varphi^{2}}=-Q,
$$

where $Q$ is the dimensionless space charge density

$$
Q=\frac{q R^{2}}{\varepsilon_{\mathrm{a}} U_{0}},
$$

where $R$ and $U_{0}$ quantities on the right-hand side of equation are dimensional; $\varepsilon_{\mathrm{a}}$ is the absolute dielectric permittivity of the semiconductor or toner, and $q$ is the dimensional space charge density $\left(\mathrm{C} / \mathrm{m}^{3}\right)$. Since in the PCT region the polar radius $\rho$ varies in a finite interval $1 \leq \rho \leq h$ (Fig. 1), and the system is symmetrical relative to the $x O z$ plane (Fig. 2), the general expression of the potential is a linear combination of partial solutions of the homogeneous equation (1):

$$
\begin{aligned}
& \text { Const }, \quad \ln \rho, \quad \rho^{n} \cos (n \cdot \varphi), \quad \rho^{-n} \cos (n \cdot \varphi), \\
& n=1,2, \ldots,
\end{aligned}
$$

plus a partial solution of the inhomogeneous equation $U_{q}$. Assuming that $Q$ is independent of both $\varphi$ and $\rho$, we obtain

$$
U_{q}=-\frac{1}{4} Q \rho^{2} .
$$

In order to derive the general expressions for potentials in the semiconductor and in the toner, we assume that there is no space charge in the semiconductor, i.e.
$U_{q}=0$. Thus, the general expression for the potential in the semiconductor is

$U_{\mathrm{S}}(\rho, \varphi)=A_{0}+A \ln \rho+\sum_{n=1}^{\infty}\left(A_{n} \rho^{-n}+a_{n} \rho^{n}\right) \cos n \varphi$,

where $A_{0}, A, A_{n}, a_{n}$ are constants. $U_{\mathrm{S}}$ must satisfy the boundary condition $U_{\mathrm{S}}(1, \varphi)=1$, so that $A_{0}=1$, $a_{n}=-A_{n}$, and (5) becomes

$$
U_{\mathrm{S}}(\rho, \varphi)=1+A \ln \rho+\sum_{n=1}^{\infty} A_{n}\left(\rho^{-n}-\rho^{n}\right) \cos n \varphi,
$$

where $1 \leq \rho \leq \rho_{1}$ (Fig. 1), $0 \leq \varphi \leq \pi$ (Fig. 2).

Since the toner space charge density $Q \neq 0$, the potential inside the toner $U_{\mathrm{T}}$ is given by

$$
\begin{aligned}
U_{\mathrm{T}}(\rho, \varphi)= & B_{0}+B \ln \rho-\frac{1}{4} Q \rho^{2} \\
& +\sum_{n=1}^{\infty}\left(B_{n} \rho^{-n}+b_{n} \rho^{n}\right) \cos n \varphi
\end{aligned}
$$

where $B_{0}, B, B_{n}$, and $b_{n}$ are constants. The boundary conditions at $\rho=\rho_{1}$ (where $\rho_{1}-1$ is the semiconductor thickness, see Fig. 1) are

$$
\begin{aligned}
& U_{\mathrm{S}}(\rho, \varphi)=U_{\mathrm{T}}(\rho, \varphi), \\
& \left.\left(\frac{\partial U_{\mathrm{S}}}{\partial \rho}-\frac{\varepsilon_{2}}{\varepsilon_{1}} \frac{\partial U_{\mathrm{S}}}{\partial \rho}\right)\right|_{\rho=\rho_{1}}=\sigma,
\end{aligned}
$$

where $\varepsilon_{2}$ and $\varepsilon_{2}$ are the relative dielectric permittivity of the semiconductor and the toner respectively, $\sigma$ is the dimensionless surface charge density on the semiconductor-toner interface

$$
\sigma=\frac{\sigma_{\mathrm{d}} R}{\varepsilon_{1 \mathrm{a}} U_{0}},
$$

with $R$ and $U_{0}$ dimensional; $\varepsilon_{1 \mathrm{a}}$ is the absolute dielectric permittivity of the semiconductor, and $\sigma_{\mathrm{d}}$ is the dimensional surface charge density, which is assumed constant.

This work uses the approximation of complete charge compensation, therefore

$$
2 \pi \rho_{1} \sigma_{\mathrm{d}}=-\pi R\left(h^{2}-\rho_{1}^{2}\right) q,
$$

where $\sigma_{\mathrm{d}}, R, q$ are dimensional quantities, and $h-\rho_{1}$ is the dimensionless thickness of the toner (Fig. 1). From (11), (10), after inserting the toner absolute dielectric permittivity $\varepsilon_{2 \mathrm{a}}$ in (2), we obtain

$$
\sigma=\frac{\varepsilon_{2}}{2 \varepsilon_{1}} \frac{h^{2}-\rho_{1}^{2}}{\rho_{1}} Q .
$$


Using relations (8) and (9), we obtain the expression for the $B_{0}$ coefficient, which appears in equation (7), via the $B$ coefficient, and the expressions for the $B_{n}$ and $b_{n}$ coefficients via the $A_{n}$ coefficient (see Appendix A). The resulting expression of the potential in the toner is

$$
\begin{aligned}
U_{\mathrm{T}}(\rho, \varphi)= & +\frac{Q}{4}\left(\rho_{1}^{2}-\rho^{2}-2 h^{2} \frac{\varepsilon_{2}}{\varepsilon_{1}} \ln \rho_{1}\right) \\
& +\left(\frac{\varepsilon_{2}}{\varepsilon_{1}} \ln \rho_{1}+\ln \frac{\rho}{\rho_{1}}\right) B \\
& +\sum_{n=1}^{\infty} A_{n}\left(\frac{\alpha_{n}}{\rho^{n}}+\beta_{n} \frac{\rho^{n}}{\rho_{1}^{2 n}}\right) \cos n \varphi,
\end{aligned}
$$

where $\rho_{1} \leq \rho \leq h, 0 \leq \varphi \leq \pi$ (Fig. 2),

$$
\begin{aligned}
& \alpha_{n}=\frac{1}{2}\left[1+\frac{\varepsilon_{2}}{\varepsilon_{1}}-\left(1-\frac{\varepsilon_{2}}{\varepsilon_{1}}\right) \rho_{1}^{2 n}\right], \\
& \beta_{n}=\frac{1}{2}\left[1-\frac{\varepsilon_{2}}{\varepsilon_{1}}-\left(1+\frac{\varepsilon_{2}}{\varepsilon_{1}}\right) \rho_{1}^{2 n}\right] .
\end{aligned}
$$

The constants $B$ and $A_{n}$ are obtained from relationships between $U_{\mathrm{T}}$ and the potential $U_{\mathrm{A}}$ of the ambient air at the point $\rho=h$.

\section{The case of infinite dielectric permittivity of paper $\left(\varepsilon_{\mathrm{p}}=\infty\right)$}

When the dielectric permittivity of the paper is infinity, the surface potential of the paper facing the toner is equal to the potential of the transfer cylinder, i.e., it is equal to zero (the paper is assumed to be infinite in the $y$ direction). The potential $U_{\mathrm{A}}$ of the air between toner and paper must satisfy the boundary condition $U_{\mathrm{A}}=0$ when $x=-h$ (Fig. 2(a)), and it also must obey the corresponding continuity conditions between $U_{\mathrm{A}}$ and $U_{\mathrm{T}}$. In order to simplify the mathematical expressions following from the boundary condition $U_{\mathrm{A}}(x=-h)=$ $0, U_{\mathrm{A}}$ is there expressed in terms of solutions of Eq. (1) in two coordinate systems with different points of origin: $O$ (as in Section 2) and $O^{\prime}$ defined by $x=-2 h$, where the latter point is obtained by reflecting $O$ in the paper surface (Fig. 2(a)). The analysed region may be at an arbitrary distance from $O$ and $O^{\prime}$, and the expression for $U_{\mathrm{A}}$ must therefore contain only terms that do not vanish at infinity, i.e.

$U_{\mathrm{A}}(\rho, \varphi)=C \ln \frac{\rho}{\rho^{\prime}}+\sum_{n=1}^{\infty}\left(C_{n} \frac{\cos n \varphi}{\rho^{n}}+D_{n} \frac{\cos n \varphi^{\prime}}{\rho^{\prime n}}\right)$.
At points $x=-h$, we have $\rho=\rho^{\prime}, \varphi=\pi-$ $\varphi^{\prime}, \cos \varphi^{\prime}=(-1)^{n} \cos n \varphi$. The boundary condition $U_{\mathrm{a}}(x=-h)=0$ is satisfied when $D_{n}=-(-1)^{n} C_{n}$, and

$$
U_{\mathrm{A}}=C \ln \frac{\rho}{\rho^{\prime}}+\sum_{n=1}^{\infty} C_{n}\left(\frac{\cos n \varphi}{\rho^{n}}+(-1)^{n} \frac{\cos n \varphi^{\prime}}{\rho^{\prime n}}\right)
$$

The radius vectors $\rho$ and $\rho^{\prime}$ at any point in space are related as follows (Fig. 2):

$$
\rho^{\prime}=r+\rho,
$$

hence these vectors and their projections on the $x$ axis are related by expressions

$$
\begin{aligned}
& \rho^{\prime}=\sqrt{r^{2}+\rho^{2}+2 r \rho \cos \varphi}, \\
& x^{\prime}=r+x=r+\rho \cos \varphi=\rho^{\prime} \cos \varphi^{\prime}, \\
& \rho^{\prime} \sin \varphi^{\prime}=\rho \sin \varphi, \\
& \varphi^{\prime}=\arccos \frac{r+\rho \cos \varphi}{\rho^{\prime}}, \quad(r=2 h) .
\end{aligned}
$$

The values of the constants $C, C_{n}, B, A_{n}$ are obtained from the conditions

$$
\left.U_{\mathrm{A}}\right|_{\rho=h}=\left.U_{\mathrm{T}}\right|_{\rho=h},\left.\quad \varepsilon_{0} \frac{\partial U_{\mathrm{A}}}{\partial \rho}\right|_{\rho=h}=\left.\varepsilon_{2} \frac{\partial U_{\mathrm{T}}}{\partial \rho}\right|_{\rho=h} .
$$

$U_{\mathrm{T}}(13)$ is expanded in a Fourier series relative to $\varphi$, so that the equations for the constants can be obtained by equating the corresponding Fourier coefficients on both sides of equalities (19). After eliminating $B$ and $A_{n}$ from the equations thus obtained, the following system of algebraic equations is obtained for the determination of $C$ and $C_{n}$ (see Appendix B):

$$
\begin{aligned}
& -\left(\frac{\varepsilon_{0}}{\varepsilon_{1}} \ln \rho_{1}+\frac{\varepsilon_{0}}{\varepsilon_{2}} \ln \frac{h}{\rho_{1}}+\ln \frac{r}{h}\right) C \\
& +\sum_{n=1}^{\infty} \frac{(-1)^{n+1}}{r^{n}} C_{n}=1+\frac{Q}{4}\left(\rho_{1}^{2}-h^{2}+2 h^{2} \ln \frac{h}{\rho_{1}}\right), \\
& \frac{(-1)^{m}}{m}\left(\frac{h^{2}}{r}\right)^{m} \frac{g_{m}+1}{g_{m}-1} C+\sum_{n=1}^{\infty}\left[\delta_{m n}+(-1)^{n} T_{m n}\right] \\
& \times C_{n}=0, \quad m=1,2, \ldots .
\end{aligned}
$$



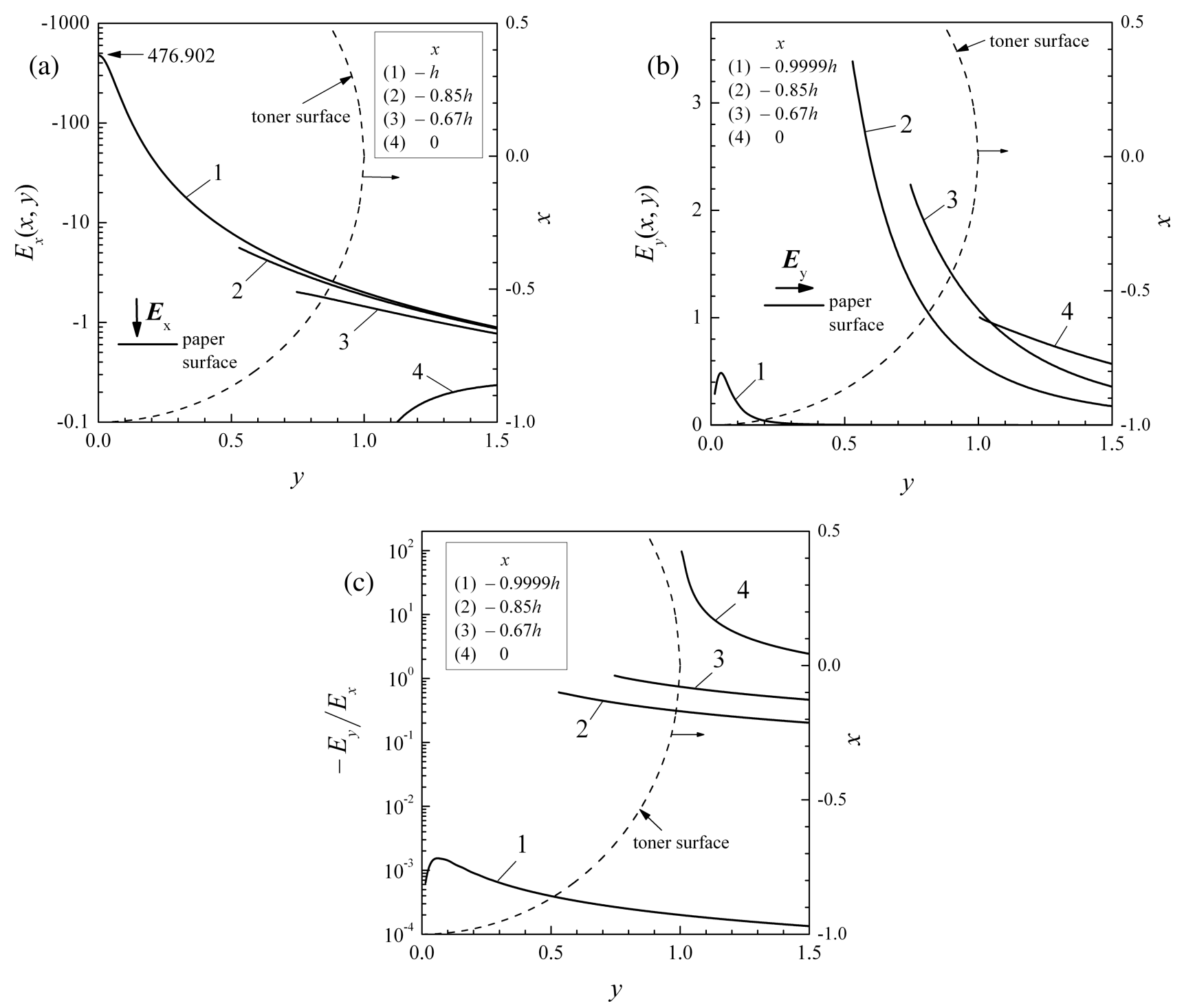

Fig. 3. Dependence of electric field strength in $U_{0}(\mathrm{~V}) / R(\mathrm{~cm})$ units on the distance $y$ in the air between toner and paper at various heights $h+x$ above the paper when $\varepsilon_{\mathrm{p}}=\infty$. Left-hand scale: in (a) vertical component (perpendicular to the paper surface), in (b) horizontal component (parallel to the paper surface), in (c) their ratio. For all curves, $h=1.005, \rho_{1}=1.001 ; \varepsilon_{1}=2 ; \varepsilon_{2}=2.5 ; Q=200$. Right-hand scale: the broken line represents the external toner surface. $y$ and $h+x$ are in $R(\mathrm{~cm})$ units.

Here,

$$
\begin{aligned}
& T_{m n}=\frac{h^{m}}{g_{m}-1} \frac{2}{\pi} \\
& \times \int_{0}^{\pi} \frac{\sin m \varphi \sin n \varphi^{\prime}-g_{m} \cos m \varphi \cos n \varphi^{\prime}}{(\sqrt{a+b \cos \varphi})^{n}} \mathrm{~d} \varphi \\
& a=r^{2}+h^{2}, \quad b=2 r h \\
& g_{m}=\frac{\varepsilon_{2}}{\varepsilon_{0}} \frac{\alpha_{m}-\beta_{m}\left(h / \rho_{1}\right)^{2 m}}{\alpha_{m}+\beta_{m}\left(h / \rho_{1}\right)^{2 m}},
\end{aligned}
$$

and $\varphi^{\prime}$ is calculated from Eq. (18) after setting $\rho=h$.
Equations (20) are solved by replacing infinite sums with finite sums: $n_{\max }=N$, the value of $N$ being chosen so that any further increase does not affect the final results within the permitted uncertainty limits. Results of the calculation of various components of the electric field strength in the air between toner and paper (perpendicular component relative to the paper surface $E_{x}(x, y)=-\partial U_{\mathrm{A}} / \partial x$, parallel component relative to the paper surface $E_{y}(x, y)=$ $-\partial U_{\mathrm{A}} / \partial y$, radial component relative to toner external surface $E_{\rho}(h, \varphi)=-\left.\left(\partial U_{\mathrm{A}} / \partial \rho\right)\right|_{\rho=h}$, and tangential component relative to toner external surface $\left.E_{\varphi}(h, \varphi)=-\left.\left(1 / \rho \cdot \partial U_{\mathrm{A}} / \partial \varphi\right)\right|_{\rho=h}\right)$ near the toner are shown in Figs. 3 and 4. In the case discussed in this section $\left(\varepsilon_{\mathrm{p}}=\infty\right)$, the results obtained do not depend 

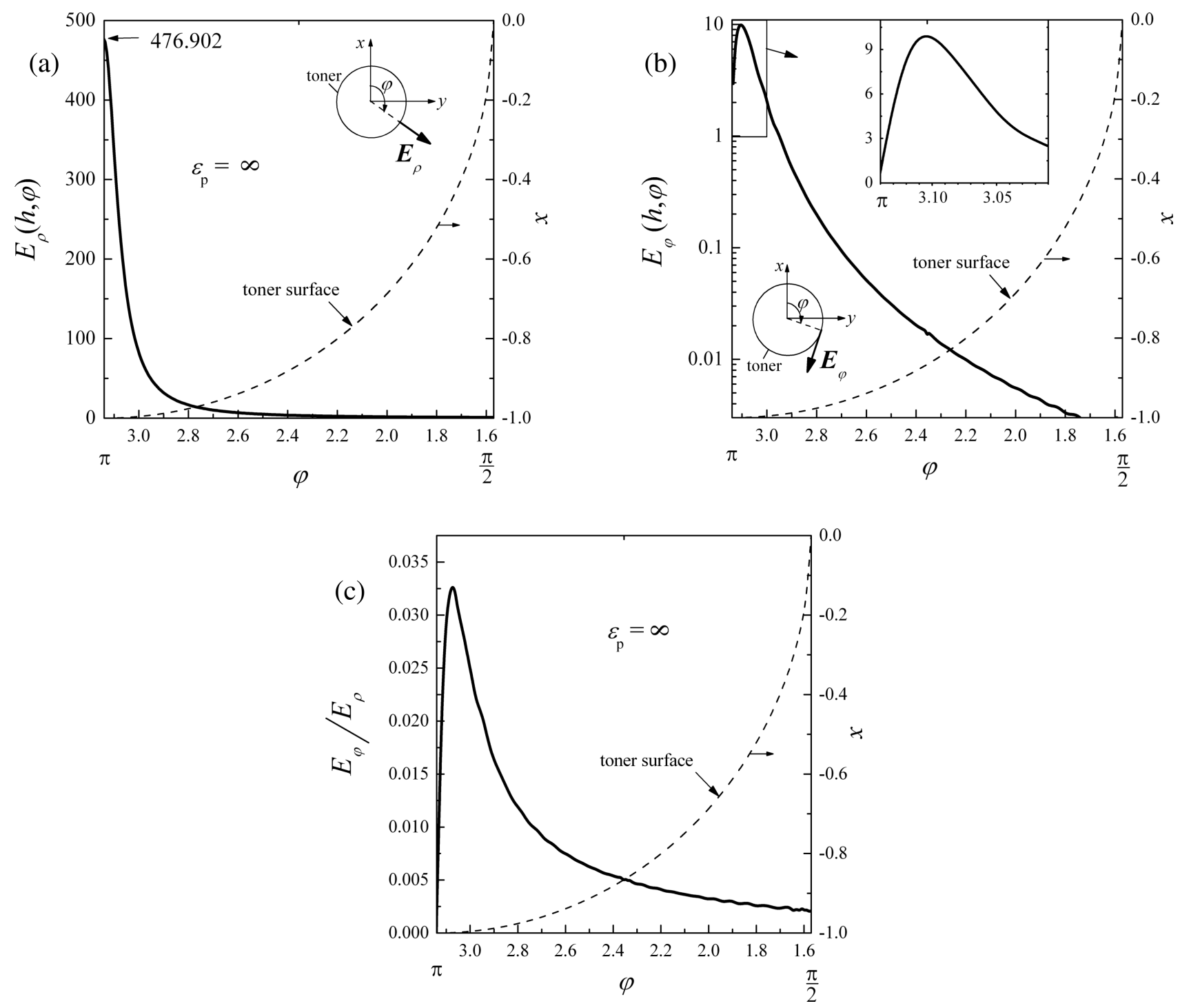

Fig. 4. Dependence of electric field strength in $U_{0}(\mathrm{~V}) / R(\mathrm{~cm})$ units on the angle $\varphi$ (Fig. 2(a)) in the air near the toner surface $(\rho=h)$ when $\varepsilon_{\mathrm{p}}=\infty$. Left-hand scale: in (a) radial component, in (b) tangential component, in (c) their ratio. $h, \rho_{1}, \varepsilon_{1}, \varepsilon_{2}, Q$ are the same as in

Fig. 3. Right-hand scale: the broken line represents the external toner surface.

on the size of the support cylinder, and the calculated value of $E_{x}$ is the largest possible value of $E_{x}$ that can be obtained by varying $\varepsilon_{\mathrm{p}}$ and paper thickness.

\section{The case $\varepsilon_{\mathrm{p}}=\varepsilon_{0}$}

Assuming hypothetically that the paper has dielectric permittivity $\varepsilon_{\mathrm{p}}=\varepsilon_{0}$ and has no space charge, the paper is mathematically equivalent to air, and the magnitude of $E_{x}$ near the toner is the smallest possible. The field strength depends on the radius of the transfer cylinder and on the paper thickness. Considering the case when transfer roller is replaced by the conductive plate, i. e. when $\rho_{2} \rightarrow \infty$ (the case of finite $\rho_{2}$ will be discussed separately), we can then use the formulas of Sec. 3, with $r$ replaced by $r=2(h+d)$. The dependence of the field strength components is shown in Figs. 5 and 6.

\section{The case of the real paper}

In a 1D system, e.g. made of an air and dielectric layer having no space charge, the electrostatic field strength in the air does not depend on dielectric thickness and dielectric permittivity separately but depends on their ratio only. In the system shown in Fig. 1 the paper and the air between paper and toner is not a 1D system but it may have some properties of 1D system locally. Having in mind that the paper thickness $d \leq$ 0.02 and obtaining the values of electric field strength at paper surface from Figs. 3 and 5, we obtain that the 

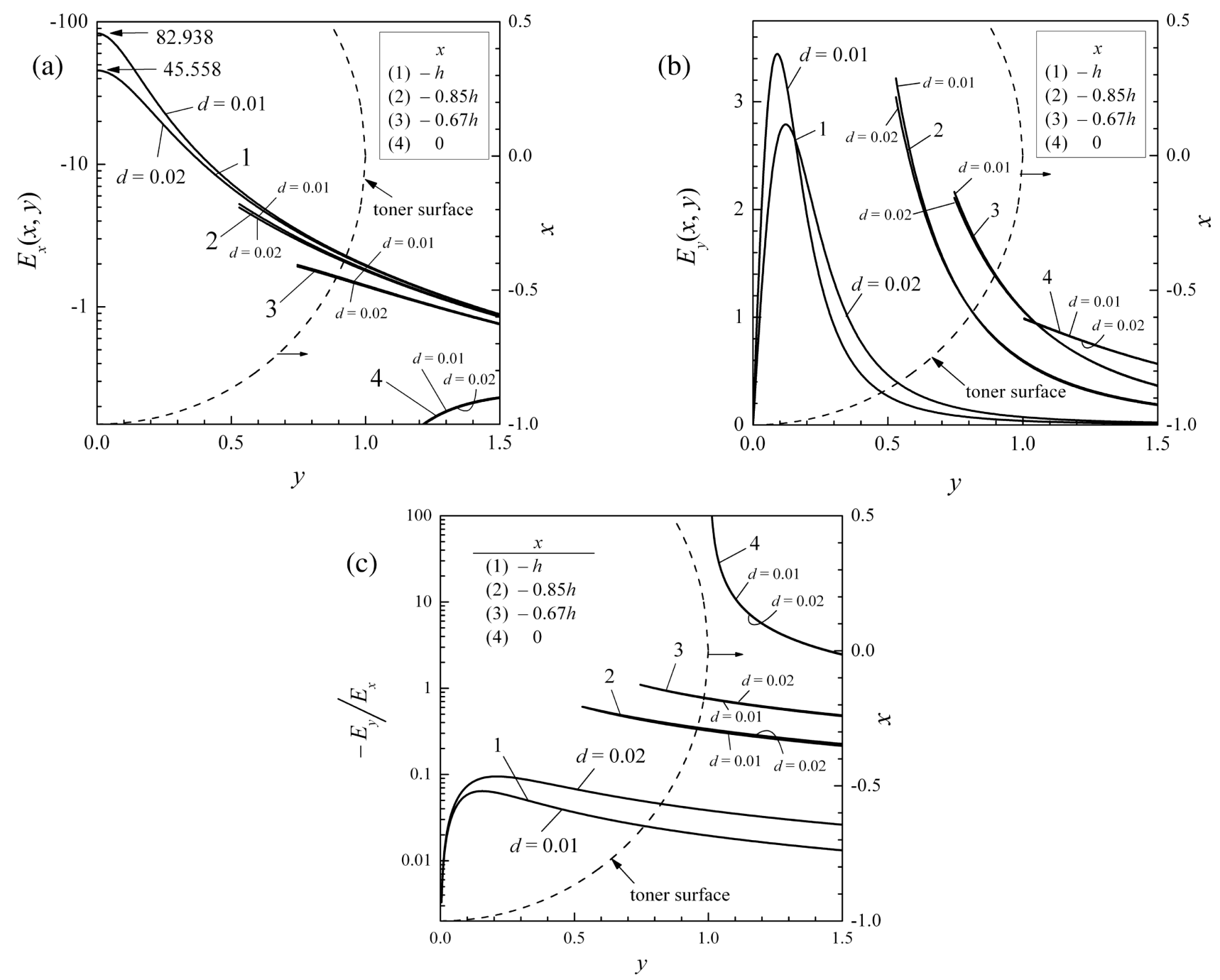

Fig. 5. Dependence of electric field strength in $U_{0}(\mathrm{~V}) / R(\mathrm{~cm})$ units on the distance $y$ in the air between toner and paper at various heights $h+x$ above the paper when $\varepsilon_{\mathrm{p}}=\varepsilon_{0}, \rho_{2} \rightarrow \infty$ for two values of paper thickness $d$. Left-hand scale: in (a) vertical component, in (b) horizontal component, in (c) their ratio. For all curves, $h=1.005, \rho_{1}=1.001 ; \varepsilon_{1}=2 ; \varepsilon_{2}=2.5 ; Q=200$. Right-hand scale: the broken line represents the external toner surface. $y$ and $h+x$ are in $R(\mathrm{~cm})$ units.

change of electric field strength in the practicaly important $y$ axis region $0.1 \leq y \leq 0.2$ does not exceed $10 \%$. Thereby the local homogeneity on $y$ axis exists at the mentioned accuracy and herewith one-dimensionality exists also. Thus in the analysis of a real paper case we consider that the paper dielectric properties are dependent only on its dielectric thickness $d_{0}=d / \varepsilon_{\mathrm{p}}$. The validity of this approach we will analyse later but here we will only note that in the limiting cases $\varepsilon_{\mathrm{p}}=\infty$, $\varepsilon_{\mathrm{p}}=\varepsilon_{0}\left(d_{0}=0, d_{0}=d\right.$ respectively $)$ the dielectric thickness approach is accurate.

At above-mentioned conditions there is no need in the additional mathematical apparatus to calculate the electric field strength: it is sufficient to replace the paper thickness $d_{0}$ by the paper dielectric thickness in the formulas from the third section $\left(\varepsilon_{\mathrm{p}}=\varepsilon_{0}\right)$. The calculation results are presented in Figs. 7-9.

\section{Discussion}

The results are discussed for a single side of the gap between the photoreceptor and the paper that corresponds to $y \geq 0$. The electric field strength and its distribution on the other side of the gap $(y \leq 0)$ will be different because in a printer the toner is then on the paper and the calculation is not directly applicable then.

The results presented in Figs. 4 and 6 indicate that the radial and tangential components $E_{\rho}$ and $E_{\varphi}$ of the electric field on the toner surface exist in an approximately 30 -degree wide interval $180^{\circ}>\varphi>150^{\circ}$ (this interval corresponds to a distance equal to 0.6 times the photoreceptor radius, as measured along the paper surface) and rapidly decrease to zero with increasing $\varphi$. When $\varepsilon_{\mathrm{p}}=\infty$, the largest values of $E_{\varphi}$ and of the ratio $E_{\varphi} / E_{\rho}$ (see Fig. 4(b, c)) are larger than the correspond- 

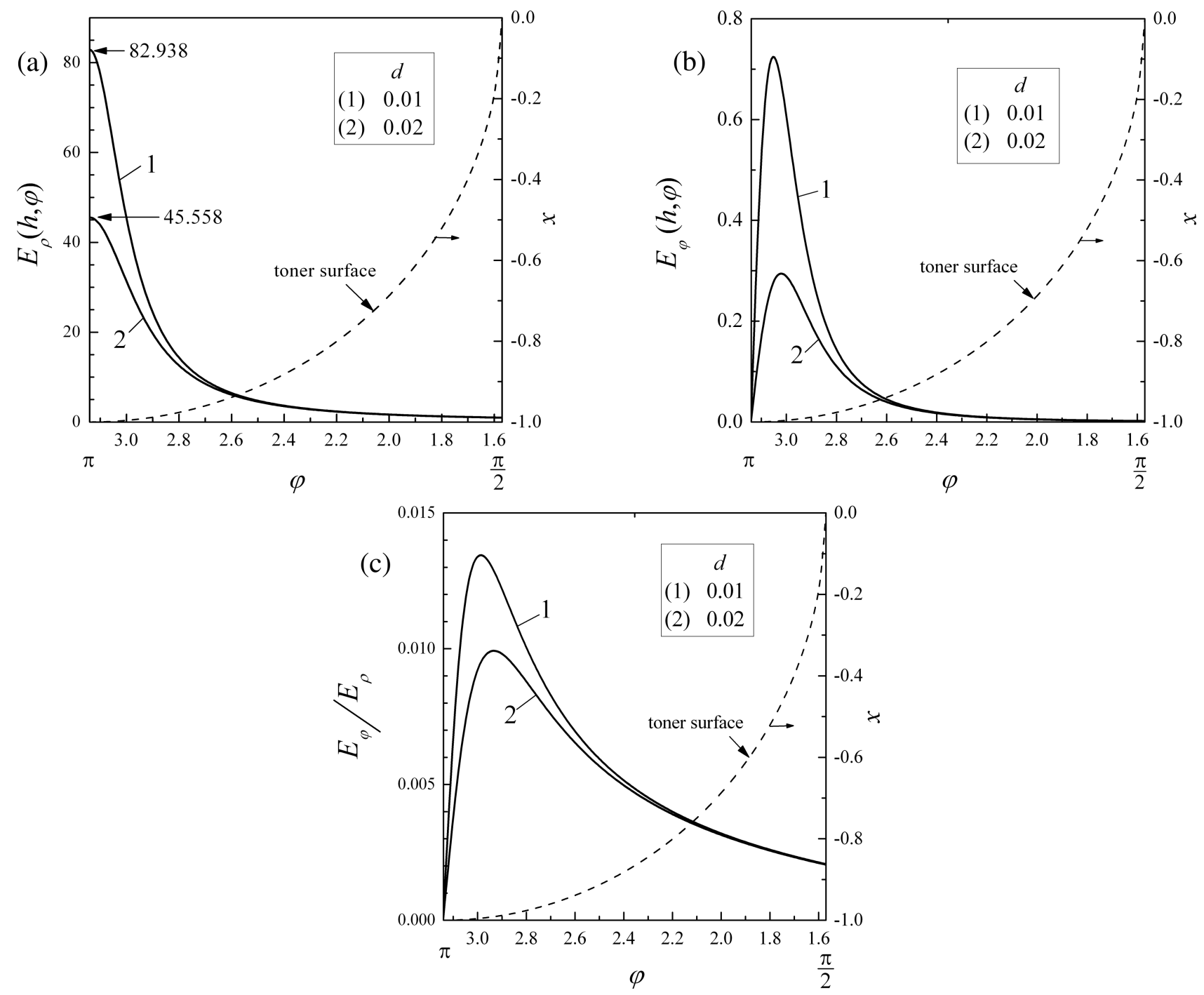

Fig. 6. Dependence of electric field strength in $U_{0}(\mathrm{~V}) / R(\mathrm{~cm})$ units on the angle $\varphi$ (Fig. 2(b)) in the air near the toner surface $(\rho=h)$ when $\varepsilon_{\mathrm{p}}=\varepsilon_{0}, \rho_{2} \rightarrow \infty$ for two values of paper thickness $d$. Left-hand scale: in (a) radial component, in (b) tangential component, in (c) their ratio. $h, \rho_{1}, \varepsilon_{1}, \varepsilon_{2}, Q$ are the same as in Fig. 5. Right-hand scale: the broken line represents the external toner surface.

ing values when $\varepsilon_{\mathrm{p}}=\varepsilon_{0}$ (Fig. 6(b,c)), and the latter values decrease with increasing paper thickness $d$. For the real paper $\varepsilon_{\mathrm{p}}>\varepsilon_{0}$, but an increase in $\varepsilon_{\mathrm{p}}$ has qualitatively the same effect as a decrease in $d$, and this means that for real paper the largest values of $E_{\varphi}$ and of the ratio $E_{\varphi} / E_{\rho}$ would not exceed the values obtained for $\varepsilon_{\mathrm{p}}=\infty$. Thus, for the analysed geometry and the $h, \rho_{1}$, $\varepsilon_{1}, \varepsilon_{2}$ values indicated in Figs. 4, 6 and for any $d$ and $\varepsilon_{\mathrm{p}}$, $E_{\varphi}$ does not exceed $0.04 E_{\rho}$ (see Fig. 4(c)). Therefore, in the mentioned case the field lines can be assumed to be perpendicular to the toner surface.

On the paper surface, the perpendicular and parallel to paper surface components $E_{x}(-h, y)$ and $E_{y}(-h, y)$ are greatest in the interval $0 \leq y \leq 0.5$ (Figs. 3(a), $5(\mathrm{a}, \mathrm{b}), 7(\mathrm{a}, \mathrm{b}))$. This corresponds to the mentioned interval of $\varphi$ values $\left(180^{\circ}>\varphi>150^{\circ}\right)$. When $\varepsilon_{\mathrm{p}}=\varepsilon_{0}$, the largest value of the $E_{y}$ component $\left(E_{y \mathrm{MAX}}\right)$ on the paper surface decreases with increasing $d$, but is equal to zero when $d=0$ (when $\varepsilon_{\mathrm{p}}=\infty$ ). It follows that $E_{y \mathrm{MAX}}$ has a maximum as a function of $d$. The results indicate that this maximum is at $d=0.005$. However, the ratio $E_{y \mathrm{MAX}} / E_{x}$ increases with $d$ and it can exceed 0.15 (Fig. 7(c)). This value is essentially higher as compared with that obtained analysing the paper roughness influence [8]. This ratio also shows that the assumption of the perpendicularity of electric field lines to the paper surface $[2,6]$ may be quite crude. Assuming that the field line is perpendicular to the paper surface and that $E_{y \mathrm{MAX}} / E_{x} \leq 0.03$, it follows that for paper with e.g. $\varepsilon_{\mathrm{p}}=10$ this would be the case if the paper thickness $d \leq 0.02$ (Fig. 8(c)). For example, if the photoreceptor diameter is $30 \mathrm{~mm}$, then the paper thickness would be 

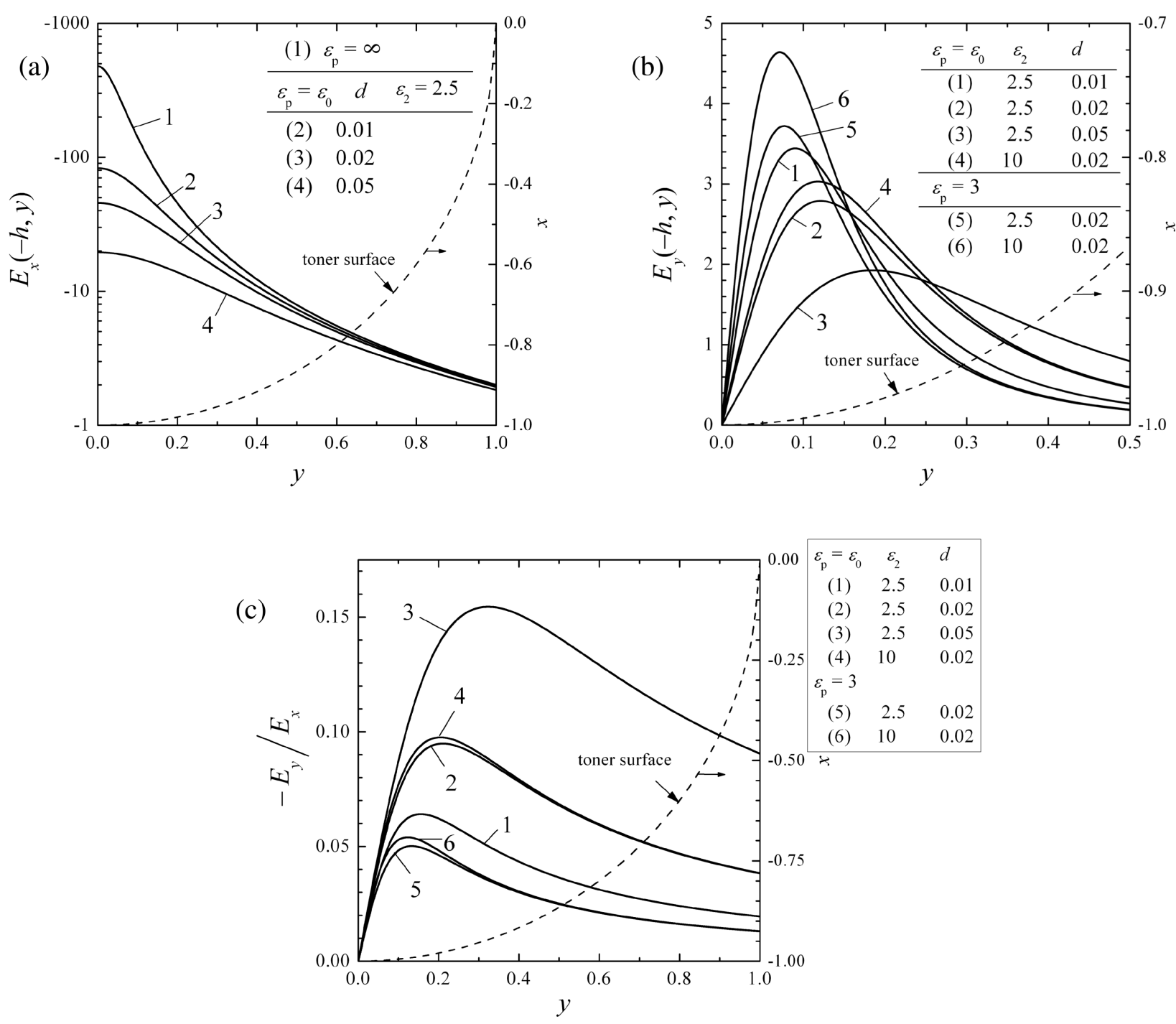

Fig. 7. Dependence of electric field strength in $U_{0}(\mathrm{~V}) / R(\mathrm{~cm})$ units on the distance $y$ near the paper surface. Left-hand scale: in (a) vertical component, in (b) horizontal component, in (c) their ratio, for several values of paper thickness and of toner and paper dielectric permittivity. For all the curves, $h=1.005, \rho_{1}=1.001 ; \varepsilon_{1}=2 ; Q=200$. Right-hand scale: the broken line represents the external toner surface. $y$ and $x$ are in $R(\mathrm{~cm})$ units.

$300 \mu \mathrm{m}$ and the paper would have a large content of salt added in order to increase the electrical conductivity. In any case, there is a real probability that the component $E_{y}$ on the paper surface decreases the resolution of an image. The non-zero value of $E_{y}$ above the paper also contributes to this effect.

An increase in toner dielectric permittivity leads to a decrease in the effective distance between the poles of potential, i. e., has the same effect as a decrease in paper thickness. It is also clear that the electric field depends on the ratio of the dielectric thicknesses of toner and paper $\left(\left(h-\rho_{1}\right) / \varepsilon_{2}\right.$ and $d / \varepsilon_{\mathrm{p}}$, respectively). For a toner thickness $h-\rho=0.004$, an increase of toner dielectric permittivity from 2.5 to 10 leads to a decrease in dielectric thickness of 0.0012. When $\varepsilon_{\mathrm{p}}=\varepsilon_{0}$ and $d=0.02$, this is an insignificant effect. However, for real paper with $\varepsilon_{\mathrm{p}}=3$ and $d=0.02$, the suggested increase in $\varepsilon_{2}$ has approximately the same effect as a change in paper thickness from $d=0.02$ to 0.0055 . When $\varepsilon_{\mathrm{p}}=\varepsilon_{0}$, $\varepsilon_{2}=2.5$ this would thus be significant. Calculations with different toner dielectric permittivity confirm these qualitative conclusions. In Fig. 7(b,c), this is shown only for the $E_{y}$ component (curves 2, 4 and 5,6).

For the model discussed here, the horizontal component of the electric field $E_{y}$ is not always zero ( $E_{y}>$ $0)$. This causes some spreading of charged toner on the paper surface, particularly in the practically important region $0<y<0.5$. Calculations (also see Fig. 7(b)) indicate that for a paper thickness $d=0.005-0.05$, the largest values of this component on the paper sur- 

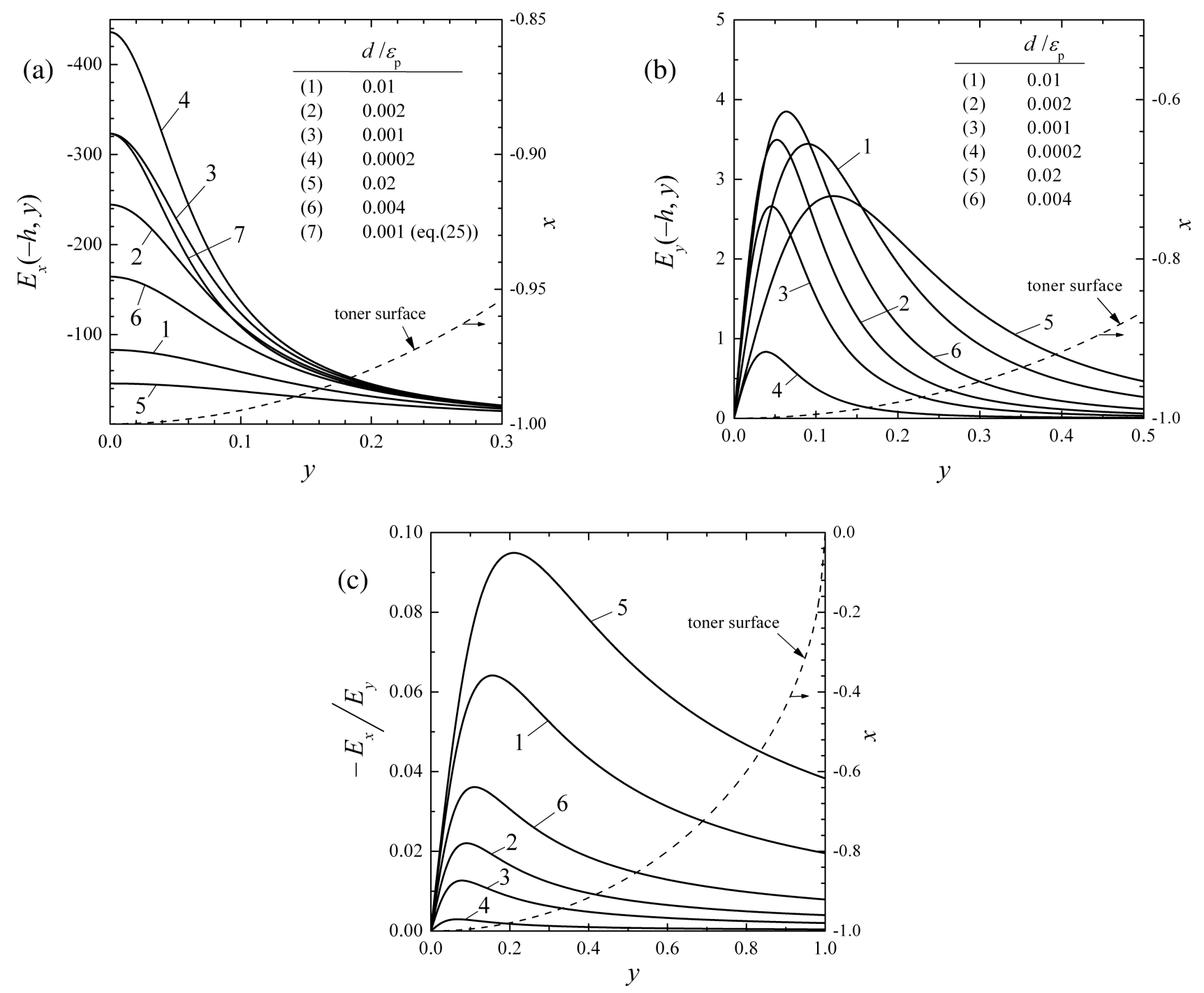

Fig. 8. Dependence of electric field strength in $U_{0}(\mathrm{~V}) / R(\mathrm{~cm})$ units on the distance $y$ near the surface of paper for different values of paper dielectric thickness $d / \varepsilon_{\mathrm{p}}$. Left-hand scale: in (a) vertical component, in (b) horizontal component, in (c) their ratio. $h, \rho_{1}, \varepsilon_{1}, Q$ are the same as in Fig. 5. $y$ and $x$ are in $R(\mathrm{~cm})$ units.

face are located between $y=0.07$ and 0.19 , which corresponds to values of $\pi-\varphi$ from $4^{\circ}$ to $11^{\circ}$. The non-zero value of $E_{y}$ in this region is the main difference between the discussed model and the 1D approximation. Another difference is the dependence of the vertical component $E_{x}$ on the distance to the paper surface (Figs. 3(a), 5(a); also see further in this section). An important contribution to $E_{y}$ comes from the logarithmic term in potential $U_{\mathrm{A}}$, which on the paper surface $(x=-h)$ is given by (Appendix B)

$$
\Delta E_{y}(-h, y)=y \frac{r(2 h-r)}{\left(h^{2}+y^{2}\right)\left[(r-h)^{2}+y^{2}\right]} C,
$$

where $C$ is the constant appearing in the expression for the potential (16). If the point $O^{\prime}$ in Fig. 2(b) is located on the axis of the support cylinder, then, for finite $\rho_{2}$, $r=h+\rho_{2}+d$. Taking into account that $C<0$ we obtain $\Delta E_{y}<0$ when $\rho_{2}+d<h$. Thus, under certain conditions the focusing of the transferred toner is possible. This indicates that using a transfer cylinder with a certain finite diameter it is possible to obtain a better image than when the toner image is transferred onto an intermediate flat transfer belt (when $\rho_{2}$ approaches infinity).

The calculated values of the vertical component of the electric field on a paper surface when $y=0$ (i.e., $\left.E_{x}(-h, 0)\right)$ are equal to the values of field strength $E$ given by the 1D model, when the 1D field is calculated under the same conditions as $E_{x}(x, y)$, i. e., when $\varepsilon_{\mathrm{p}}=$ $\infty$ or $\varepsilon_{\mathrm{p}}=\varepsilon_{0}$. This should also apply for the real paper $\left(\varepsilon_{\mathrm{p}} \neq \varepsilon_{0}\right)$. This means that the interpolated value of $E_{x}$ on the real paper surface $E_{x}^{(\mathrm{INT})}(-h, y)$ can be obtained 

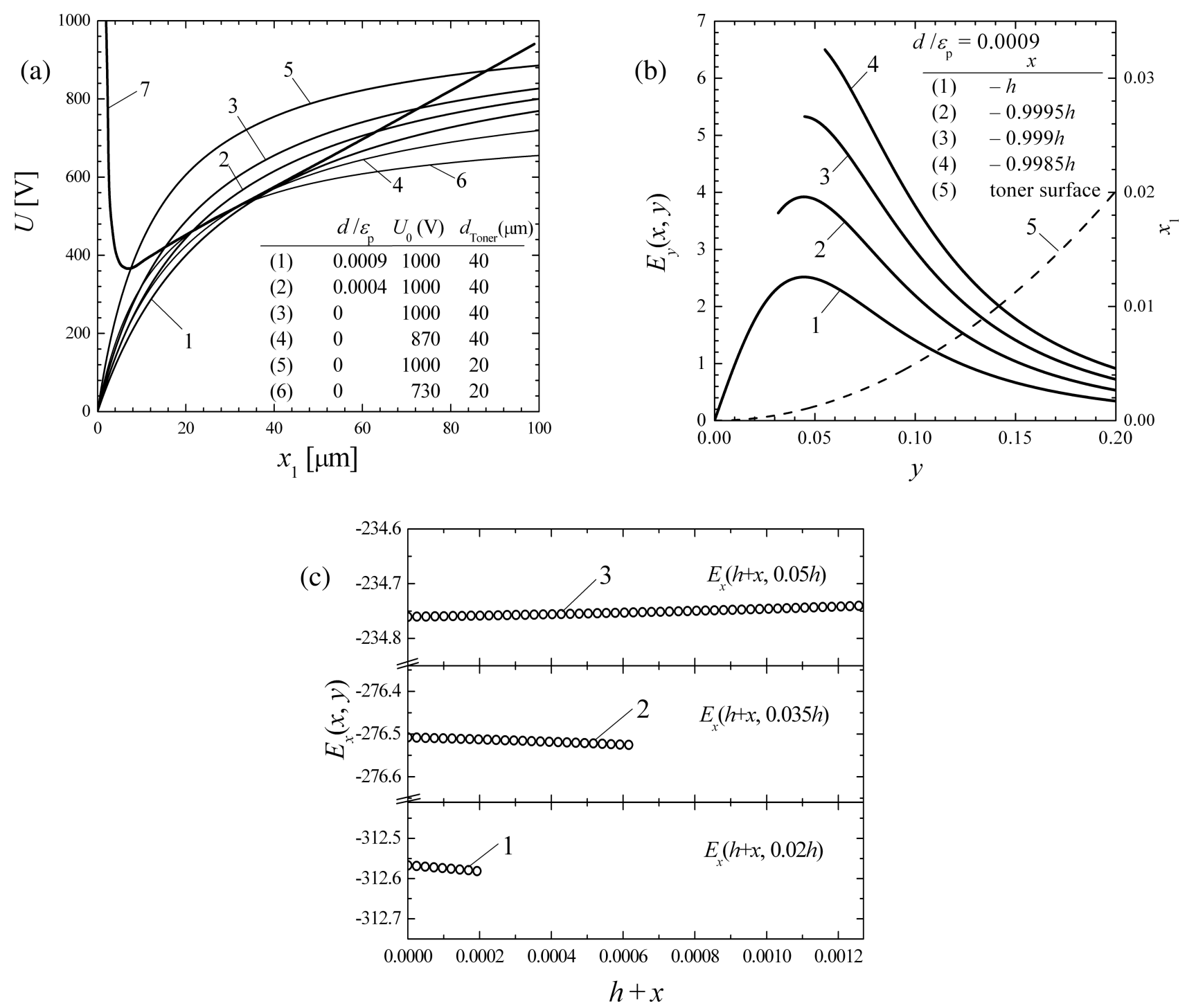

Fig. 9. Paschen effect. (a) conditions for the Paschen effect. $1, \ldots, 6$ are dependences of potential change in the air gap on the layer thickness $x_{1}$, when the cylinder radius is $1 \mathrm{~cm}$, semiconductor and toner thicknesses are 10 and $40 \mu \mathrm{m}$, respectively, dielectric permittivities are $\varepsilon_{1}=$ 2 and $\varepsilon_{2}=2.5$, respectively; 7 is Paschen curve [9]. In (b), left-hand scale is variation of the horizontal component of electric field $E_{y}$ in the Paschen effect region, right-hand scale is dependence of thickness of the air gap between paper and toner on the air coordinate $y$ (dashed line). (c) Variation of the vertical component $E_{x}$ in the Paschen effect region (the end-points of the curves correspond to the toner surface).

$$
E_{x}, E_{y} \text { are in } U_{0}(\mathrm{~V}) / R(\mathrm{~cm}) \text { units; } y, x, h+x \text { are in } R(\mathrm{~cm}) \text { units. }
$$

using the maximum value $E_{x}^{(\mathrm{MAX})}(-h, y)$ when $\varepsilon_{\mathrm{p}}=$ $\infty$ and the minimum value $E_{x}^{(\mathrm{MIN})}(-h, y)$ when $\varepsilon_{\mathrm{p}}=$ $\varepsilon_{0}$.

Calculating $E$ for real paper from the 1D equality

$$
E=-\frac{1+\frac{Q}{2}\left(h-\rho_{1}\right)^{2}}{\frac{\rho_{1}-1}{\varepsilon_{1}}+\frac{h-\rho_{1}}{\varepsilon_{2}}+\frac{d}{\varepsilon_{\mathrm{p}}}},
$$

assuming the same weights for $E_{x}^{(\mathrm{MAX})}(-h, y)$ and
$E_{x}^{(\mathrm{MIN})}(-h, y)$ for all $y$, and taking those weights from the case when $y=0$, we obtain

$$
\begin{aligned}
& E_{x}^{(\mathrm{INT})}(-h, y)=E_{x}^{(\mathrm{MAX})}(-h, y) \\
& -\frac{E_{x}^{(\mathrm{MAX})}(-h, 0)-E}{E_{x}^{(\mathrm{MAX})}(-h, 0)-E_{x}^{(\mathrm{MIN})}(-h, 0)} \\
& \times\left[E_{x}^{(\mathrm{MAX})}(-h, y)-E_{x}^{(\mathrm{MIN})}(-h, y)\right] .
\end{aligned}
$$

Figure 8(a) (curves 7 and 3) presents the comparison of the calculation results obtained by using expression (25) and by applying the paper dielectric thickness 
approximation. The proximity of the results shows the reliability of the used methods.

It is known (e. g. [2,7]) and has been mentioned in the introduction of this work that one of the transferred toner image quality deteriorative factors on the Paschen discharge. The conditions of the latter effect are not investigated enough. Our work gives the possibility to determine the initial conditions of Paschen effect in the toner image transfer nip. Without going further into the air ionization phenomena mechanisms, we have taken the Paschen curve [13] which has been determined under conditions when the field strength is the same at all points in the air gap. Although in the analysed image transfer system the electric field in the air gap is not strictly homogeneous, in the supposed region of Paschen effect $y$ does not exceed $0.15 R(\mathrm{~cm})$ and the degree of inhomogeneity as seen from Fig. $9(b, c)$ is not significant.

Figure 9 presents calculation results when the paper is characterized by its dielectric thickness $d_{0}$. Figure 9(a) illustrates the Paschen effect and shows that at $U_{0}=10^{3} \mathrm{~V}, R=0.01 \mathrm{~m}$ the semiconductor thickness $\rho_{1}-R$ is $10 \mu \mathrm{m}$ and toner thickness $h-\rho_{1}$ is $40 \mu \mathrm{m}$, and the largest interval of thickness $x_{1}$ of the air gap between the paper and the toner where the effect is possible is from 16 to $73 \mu \mathrm{m}$. This interval corresponds to a paper dielectric thickness $d_{0}=0$. As the dielectric thickness increases, the interval for the effect narrows and, when $d_{0} \geq 9 \mu \mathrm{m}$, it disappears completely. The thickness $x_{1}$ and its air coordinate $y$ are related to each other by the expression $y=\sqrt{\left(2 h-x_{1}\right) x_{1}}$ (see Fig. 9(b), right-hand scale), from which it follows that for the chosen set of transfer nip parameters the abovementioned interval of $x_{1}$ values (16 to $\left.73 \mu \mathrm{m}\right)$ corresponds to $y$ values from 570 to $1210 \mu \mathrm{m}$. Calculations, which are partly illustrated in Fig. 9(b), indicate that when the paper dielectric thickness varies from 2 to $200 \mu \mathrm{m}$, the $y$ coordinate, which gives the distance from the $x O z$ plane to the point of the maximum horizontal component of the electric field strength $E_{y}$ (i.e., $E_{y \mathrm{MAX}}$ ), increases from 390 to $1210 \mu \mathrm{m}$. Thus, it includes the complete interval of the maximum Paschen effect. Besides, $E_{y \mathrm{MAX}}$ increases from the paper surface towards the toner surface. For example when the paper dielectric thickness $d_{0}=40 \mu \mathrm{m}$, $E_{y \mathrm{MAX}}$ increases from 3.8 up to $5.3 \mathrm{~V}$ and comprises $3-$ $4 \%$ of the vertical component of field strength in that region of the air (see Fig. 8). In the region of the Paschen effect, the vertical component of field strength $E_{x}$ does not change significantly from the paper surface towards the toner (see Fig. 9(c)) and it is practically the same as in the 1D model, which assumes the paper thickness equal to $x_{1}$. Far from the Paschen effect region, e.g., when $y \geq 0.005 \mathrm{~m}$, the relative increase in $E_{x}$ from the paper towards the toner is more noticeable (see Figs. 3(a), 5(a)).

At the mentioned values of $R, \rho_{1}$, and $h$ the Paschen effect can only occur when the cylinder potential exceeds $870 \mathrm{~V}$ (see Fig. 9(a)). However, in the case of paper with $\varepsilon_{\mathrm{p}}=10$ and thickness $100 \mu \mathrm{m}$ (dielectric thickness 0.001) the Paschen effect will occur when the potential exceeds $1020 \mathrm{~V}$. The situation is different for other values of $R, \rho_{1}$, and $h$. If $R$ and $\rho_{1}$ are the same as before and the toner thickness is reduced by one half, then the Paschen effect takes place when $U_{0}$ exceeds $730 \mathrm{~V}$, whereas the maximum region of this effect widens (curve 5 in Fig. 9(a)) and its distance to the $x O z$ plane ranges from 390 to $1330 \mu \mathrm{m}$. According to the 1D model [7], at $U_{0}=1000 \mathrm{~V}$, paper thickness $80 \mu \mathrm{m}$, toner thickness $13.2 \mu \mathrm{m}$, and paper and toner relative dielectric permittivity 2.5 and 1.7 respectively, the Paschen effect begins at air gap $18.6 \mu \mathrm{m}$. Our calculation shows that in the case of work [7] parameters and when $R=1 \mathrm{~cm}$ the Paschen effect is impossible. For the effect at abovementioned air gap to occur the potential must be increased up to $\geq 1.5 U_{0}$. Calculation also shows that the Paschen effect potential and the components of electric field in the air between the toner and the paper depend (accuracy $\leq 1 \%$ ) only on the ratio $d_{\text {toner }} / \varepsilon_{2}$ and do not depend on either the toner thickness or its dielectric permittivity separately.

For other values of $R$, e.g. when $R$ varies from 0.5 to $2 \mathrm{~cm}$, the minimum value of the potential which must be exceeded in order for the Paschen effect to occur remains equal to $870 \mathrm{~V}$, whereas the distance from the $x O z$ plane to the maximum region of the effect increases and this region becomes wider (when $R=2 \mathrm{~cm}$, the furthest point is at $1710 \mu \mathrm{m}$ ).

\section{Conclusions}

Exact solutions of the Poisson equation for 2D case of a flat paper transported in a toner image transfer system allow one to draw the following conclusions:

1. With the photoreceptor cylinder radius $R=0.01 \mathrm{~m}$, the semiconductor thickness $10 \mu \mathrm{m}$, and the toner thickness $40 \mu \mathrm{m}$, the Paschen effect occurs when the cylinder potential $U_{0}$ exceeds $870 \mathrm{~V}$. When $U_{0}=10^{3} \mathrm{~V}$, the distances from the $x O z$ plane (i.e., the plane perpendicular to the paper surface passing through the axis of the cylinder) to the end-points 
of the maximum interval where the Paschen effect takes place are 570 and $1210 \mu \mathrm{m}$. As the paper dielectric thickness $d_{0}$ increases from $d_{0}=0$ (i.e., from $\varepsilon_{\mathrm{p}}=\infty$ to finite $\varepsilon_{\mathrm{p}}$ ), the interval of the effect narrows, and it disappears completely when $d_{0} \geq$ $9 \mu \mathrm{m}$ (Fig. 9).

2. In the air between the paper and toner and on the paper surface, the electric field strength has a horizontal component parallel to the paper surface, $E_{y}$, the maximum $E_{y \mathrm{MAX}}$ of which at paper surface occurs at a distance from the $x O z$ plane that depends on the paper dielectric thickness: as the dielectric thickness increases from 2 to $200 \mu \mathrm{m}$, the distance increases from 390 to $1210 \mu \mathrm{m}$ (Fig. 8). In the region of the Paschen effect, the value of $E_{y \mathrm{MAX}}$ can be up to $4 \%$ of the value of the vertical component of field strength perpendicular to the paper surface. At the paper dielectric thickness $d_{0}>200 \mu \mathrm{m}$ and outside of the Paschen effect region the horizontal component parallel to the paper surface can be even $10-15 \%$ of the vertical component (Figs. 7 and 8 ).

3. The perpendicular to the paper surface component of the electric field strength $E_{x}$ rapidly decreases with increasing distance from the $x O z$ plane. In the region between the $x O z$ plane and the furthest edge of the Paschen effect region, the dependence of $E_{x}$ on the distance to the paper surface in the nip between the paper and the toner is insignificant and that component is practically equal to the field strength obtained in the 1D model (Fig. 9(c)). However when the distance from $x O z$ plane outside the Paschen effect furthest edge is increasing, the calculated in a 2D model $E_{x}$ value depends substantially (Figs. 3 and 5) on the distance from the paper surface and differs essentially from the value obtained in 1D model.

\section{Appendix}

\section{A. Derivation of expression (13) for toner potential}

The expanded versions of equations (8) and (9) are

$$
1+A \ln \rho_{1}+\sum_{n=1}^{\infty} A_{n} \frac{1-\rho_{1}^{2 n}}{\rho_{1}^{n}} \cos n \varphi=B_{0}+
$$

$$
\begin{aligned}
& +B \ln \rho_{1}-\frac{1}{4} Q \rho_{1}^{2}+\sum_{n=1}^{\infty} \frac{B_{n}+\rho_{1}^{2 n} b_{n}}{\rho_{1}^{n}} \cos n \varphi,(\mathrm{A},) \\
& \frac{A}{\rho_{1}}-\sum_{n=1}^{\infty} A_{n} n \frac{1+\rho_{1}^{2 n}}{\rho_{1}^{n+1}} \cos n \varphi=\frac{\varepsilon_{2}}{\varepsilon_{1}}\left(\frac{B}{\rho_{1}}-\frac{1}{2} Q \rho_{1}\right. \\
& \left.+\sum_{n=1}^{\infty} n \frac{b_{n} \rho_{1}^{2 n}-B_{n}}{\rho_{1}^{n+1}} \cos n \varphi-\frac{h^{2}-\rho_{1}^{2}}{2 \rho_{1}} Q\right) .
\end{aligned}
$$

In the latter equation, the expression of $\sigma$ given in equation (12) has been incorporated. After equating the coefficients at $\cos n \varphi$ and the terms that do not depend on $\varphi$ on both sides of the equations, we obtain

$$
\begin{aligned}
& 1+A \ln \rho_{1}=B_{0}+B \ln \rho_{1}-\frac{1}{4} Q \rho_{1}^{2}, \\
& A=\frac{\varepsilon_{2}}{\varepsilon_{1}}\left(B-\frac{1}{2} Q h^{2}\right), \\
& A_{n}\left(1-\rho_{1}^{2 n}\right)=B_{n}+\rho_{1}^{2 n} b_{n}, \\
& A_{n}\left(1-\rho_{1}^{2 n}\right) \frac{\varepsilon_{1}}{\varepsilon_{2}}=B_{n}-\rho_{1}^{2 n} b_{n} .
\end{aligned}
$$

From (A.3) it follows that

$B_{0}=1+\frac{Q}{4}\left(\rho_{1}^{2}-2 \frac{\varepsilon_{2}}{\varepsilon_{1}} h^{2} \ln \rho_{1}\right)+\left(\frac{\varepsilon_{2}}{\varepsilon_{1}}-1\right) \ln \rho_{1} B$.

From (A.4),

$$
\begin{aligned}
& B_{n}=\frac{1}{2}\left[1+\frac{\varepsilon_{1}}{\varepsilon_{2}}-\left(1-\frac{\varepsilon_{1}}{\varepsilon_{2}}\right) \rho_{1}^{2 n}\right] A_{n}, \\
& b_{n}=\frac{1}{2 \rho_{1}^{2 n}}\left[1-\frac{\varepsilon_{1}}{\varepsilon_{2}}-\left(1+\frac{\varepsilon_{1}}{\varepsilon_{2}}\right) \rho_{1}^{2 n}\right] A_{n} .
\end{aligned}
$$

After combining (7) with (A.5) and (A.6), equation (13) is obtained:

$$
\begin{aligned}
U_{\mathrm{T}}(\rho, \varphi)=1 & +\frac{Q}{4}\left(\rho_{1}^{2}-\rho^{2}-2 h^{2} \frac{\varepsilon_{2}}{\varepsilon_{1}} \ln \rho_{1}\right) \\
& +\left(\frac{\varepsilon_{2}}{\varepsilon_{1}} \ln \rho_{1}+\ln \frac{\rho}{\rho_{1}}\right) B \\
& +\sum_{n=1}^{\infty} A_{n}\left(\frac{\alpha_{n}}{\rho^{n}}+\beta_{n} \frac{\rho^{n}}{\rho_{1}^{2 n}}\right) \cos n \varphi .
\end{aligned}
$$




\section{B. The case $\varepsilon_{\mathrm{p}}=\infty$. Derivation of equations for calculating air potential constants and expressions for components of electric field strength}

After expansion, the conditions in equation (19) can be written as follows:

$$
\begin{aligned}
& C \ln \frac{h}{\sqrt{q+b \cos \varphi}}+\sum_{n=1}^{\infty} C_{n}\left(\frac{\cos n \varphi}{h^{n}}-(-1)^{n} \frac{\cos n \varphi^{\prime}}{\sqrt{a+b \cos \varphi}}\right)=1+\frac{Q}{4}\left(\rho_{1}^{2}-h^{2}-2 \frac{\varepsilon_{2}}{\varepsilon_{1}} h^{2} \ln \rho_{1}\right) \\
& +\left(\frac{\varepsilon_{2}}{\varepsilon_{1}} \ln \rho_{1}+\ln \frac{h}{\rho_{1}}\right) B+\sum_{n=1}^{\infty} A_{n} \frac{\alpha_{n}+\left(h / \rho_{1}\right)^{2 n} \beta_{n}}{h^{n}} \cos n \varphi, \\
& C \frac{r}{h} \frac{r+h \cos \varphi}{a+b \cos \varphi}-\sum_{n=1}^{\infty} C_{n}\left[n \frac{\cos n \varphi}{h^{n+1}}-(-1)^{n} n \frac{\cos n \varphi^{\prime}-r \frac{\cos (n+1) \varphi^{\prime}}{\sqrt{a+b \cos \varphi}}}{h(\sqrt{a+b \cos \varphi})^{n}}\right]= \\
& \frac{\varepsilon_{2}}{\varepsilon_{0}}\left[-\frac{1}{2} Q h+\frac{B}{h}+\sum_{n=1}^{\infty} n A_{n} \frac{\left(\frac{h}{\rho_{1}}\right)^{2 n} \beta_{n}-\alpha_{n}}{h^{n+1}} \cos n \varphi\right]
\end{aligned}
$$

where

$$
a=r^{2}+h^{2}, \quad b=2 r h, \quad \varphi^{\prime}=\arccos \frac{r+h \cos \varphi}{\sqrt{a+b \cos \varphi}} .
$$

The final term on the left-hand side of (B.2) has been obtained using the expression

$$
\frac{\partial}{\partial \rho} \frac{\cos n \varphi^{\prime}}{\rho^{\prime n}}=-n \frac{\sin n \varphi^{\prime}}{\rho^{\prime n}} \frac{\partial \varphi^{\prime}}{\partial \rho}-n \frac{\cos n \varphi^{\prime}}{\rho^{\prime n+1}} \frac{\partial \rho^{\prime}}{\partial \rho}=-\frac{n}{\rho \rho^{\prime n}}\left[\cos n \varphi^{\prime}-r \frac{\cos (n+1) \varphi^{\prime}}{\rho^{\prime}}\right],
$$

where $\rho=h$. The second equation of (B.4) is obtained using equations (18) for $\varphi^{\prime}$ and $\rho^{\prime}$ when calculating the partial derivatives $\partial \varphi^{\prime} / \partial \rho, \partial \rho^{\prime} / \partial \rho$ and replacing $\cos \varphi$ and $\sin \varphi$ with $\cos \varphi^{\prime}$ and $\sin \varphi^{\prime}$ according to equation (18).

In order to derive the relations of Fourier coefficients that follow from (B.1) and (B.2), the relations between the free coefficients are obtained by calculating the following integral of both sides of each equation:

$$
\frac{1}{\pi} \int_{0}^{\pi} \ldots \mathrm{d} \varphi
$$

Using the 4.224.9 formula given in [10], we obtain

$$
\frac{1}{\pi} \int_{0}^{\pi} \ln \sqrt{a+b \cos \varphi} \mathrm{d} \varphi=\ln r,
$$

and the formula 2.554 .2 presented in [10] gives

$$
\frac{1}{\pi} \int_{0}^{\pi} \frac{r+h \cos \varphi}{a+b \cos \varphi} \mathrm{d} \varphi=\frac{1}{r} .
$$


Equation (B.1) then leads to

$$
C \ln \frac{h}{r}-\sum_{n=1}^{\infty} C_{n}(-1)^{n} \frac{1}{\pi} \int_{0}^{\pi} \frac{\cos n \varphi}{(\sqrt{a+b \cos \varphi})^{n}} \mathrm{~d} \varphi=1+\frac{Q}{4}\left(\rho_{1}^{2}-h^{2}-2 \frac{\varepsilon_{2}}{\varepsilon_{1}} h^{2} \ln \rho_{1}\right)+\left(\frac{\varepsilon_{2}}{\varepsilon_{1}} \ln \rho_{1}+\ln \frac{h}{\rho_{1}}\right) B,
$$

and (B.2) gives

$$
C+\sum_{n=1}^{\infty} n C_{n}(-1)^{n} \frac{1}{\pi} \int_{0}^{\pi} \frac{\cos n \varphi^{\prime}-r \frac{\cos (n+1) \varphi^{\prime}}{\sqrt{a+b \cos \varphi}}}{(\sqrt{a+b \cos \varphi})^{n}} \mathrm{~d} \varphi=\frac{\varepsilon_{2}}{\varepsilon_{0}}\left(B-\frac{1}{2} Q h^{2}\right) .
$$

After obtaining $B$ from (B.8) and substituting that expression in (B.7), we have

$$
\begin{aligned}
& -\left(\frac{\varepsilon_{0}}{\varepsilon_{1}} \ln \rho_{1}+\frac{\varepsilon_{0}}{\varepsilon_{2}} \ln \frac{h}{\rho_{1}}+\ln \frac{r}{h}\right) C \\
& +\sum_{n=1}^{\infty} C_{n}(-1)^{n+1} \frac{1}{\pi} \int_{0}^{\pi}\left[\frac{\cos \varphi^{\prime}}{(\sqrt{a+b \cos \varphi})^{n}}+n\left(\frac{\varepsilon_{0}}{\varepsilon_{1}} \ln \rho_{1}+\frac{\varepsilon_{0}}{\varepsilon_{2}} \ln \frac{h}{\rho_{1}}\right) \frac{\cos n \varphi^{\prime}-r \frac{\cos (n+1) \varphi^{\prime}}{\sqrt{a+b \cos \varphi}}}{(\sqrt{a+b \cos \varphi})^{n}}\right] \mathrm{d} \varphi= \\
& 1+\frac{Q}{4}\left(\rho_{1}^{2}-h^{2}+2 h^{2} \ln \frac{h}{\rho_{1}}\right) .
\end{aligned}
$$

Using equations (18), we transform the integrand of the (B.9) integral:

$$
\begin{gathered}
\frac{\cos n \varphi^{\prime}-r \frac{\cos (n+1) \varphi^{\prime}}{\sqrt{a+b \cos \varphi}}}{(\sqrt{a+b \cos \varphi})^{n}}=\frac{\cos n \varphi^{\prime}}{(\sqrt{a+b \cos \varphi})^{n}}-\frac{r\left(\cos n \varphi^{\prime} \cos \varphi^{\prime}-\sin n \varphi^{\prime} \sin \varphi^{\prime}\right)}{(\sqrt{a+b \cos \varphi})^{n+1}}= \\
\frac{\cos n \varphi^{\prime}}{(\sqrt{a+b \cos \varphi})^{n}}-\frac{r}{(\sqrt{a+b \cos \varphi})^{n+2}}\left[\cos n \varphi^{\prime}(r+h \cos \varphi)-h \sin n \varphi^{\prime} \sin \varphi\right]= \\
\frac{\left(h^{2}+h r \cos \varphi\right) \cos n \varphi^{\prime}}{(\sqrt{a+b \cos \varphi})^{n+2}}+r h \frac{\sin n \varphi^{\prime} \sin \varphi}{(\sqrt{a+b \cos \varphi})^{n+2}} .
\end{gathered}
$$

After integrating the final term of this equation by parts and using the following expression (which follows from equation (18)),

$$
\frac{\partial \varphi^{\prime}}{\partial \varphi}=\frac{h^{2}+h r \cos \varphi}{a+b \cos \varphi}
$$

we have

$$
r h \frac{1}{\pi} \int_{0}^{\pi} \frac{\sin n \varphi^{\prime} \sin \varphi}{(\sqrt{a+b \cos \varphi})^{n+2}} \mathrm{~d} \varphi=-\frac{1}{\pi} \int_{0}^{\pi} \frac{\left(h^{2}+r h \cos \varphi\right) \cos n \varphi^{\prime}}{(\sqrt{a+b \cos \varphi})^{n+2}} \mathrm{~d} \varphi
$$

Thus,

$$
\frac{1}{\pi} \int_{0}^{\pi} \frac{\cos n \varphi^{\prime}-r \frac{\cos (n+1) \varphi^{\prime}}{\sqrt{a+b \cos \varphi}}}{(\sqrt{a+b \cos \varphi})^{n}} \mathrm{~d} \varphi=0
$$


hence,

$$
\frac{1}{\pi} \int_{0}^{\pi} \frac{\cos (n+1) \varphi^{\prime}}{(\sqrt{a+b \cos \varphi})^{n+1}} \mathrm{~d} \varphi=\frac{1}{r} \frac{1}{\pi} \int_{0}^{\pi} \frac{\cos n \varphi^{\prime}}{(\sqrt{a+b \cos \varphi})^{n}} \mathrm{~d} \varphi .
$$

When $n=1$, the integral on the right-hand side of (B.13) (without the multiplier $1 / r$ ) is equal to (B.6). Therefore,

$$
\frac{1}{\pi} \int_{0}^{\pi} \frac{\cos n \varphi^{\prime}}{(\sqrt{a+b \cos \varphi})^{n}} \mathrm{~d} \varphi=\frac{1}{r^{n}}
$$

Hence, (B.9) coincides with the first equation of the system of equations (20).

The other equations are obtained by calculating the following integrals of both sides of equations (B.1) and (B.2):

$$
\frac{2}{\pi} \int_{0}^{\pi} \ldots \cos m \varphi \mathrm{d} \varphi, \quad m=1,2, \ldots
$$

(B.1) gives

$-C \frac{1}{\pi} \int_{0}^{\pi} \cos m \varphi \ln (a+b \cos \varphi) \mathrm{d} \varphi+\frac{C_{m}}{h^{m}}+\sum_{n=1}^{\infty} C_{n}(-1)^{n+1} \frac{2}{\pi} \int_{0}^{\pi} \frac{\cos n \varphi^{\prime} \cos m \varphi}{(\sqrt{a+b \cos \varphi})^{n}} \mathrm{~d} \varphi=\frac{\alpha_{m}+\left(h / \rho_{1}\right)^{2 m} \beta_{m}}{h^{m}} A_{m}$,

and (B.2) gives

$$
\begin{aligned}
& C r \frac{2}{\pi} \int_{0}^{\pi} \frac{r+h \cos \varphi}{a+b \cos \varphi} \cos m \varphi \mathrm{d} \varphi-\frac{m}{h^{m}} C_{m}+\sum_{n=1}^{\infty} C_{n}(-1)^{n} n \frac{2}{\pi} \int_{0}^{\pi} \frac{\cos n \varphi^{\prime}-r \frac{\cos (n+1) \varphi^{\prime}}{\sqrt{a+b \cos \varphi}}}{(\sqrt{a+b \cos \varphi})^{n}} \cos m \varphi \mathrm{d} \varphi= \\
& \frac{\varepsilon_{2}}{\varepsilon_{0}} \frac{m}{h^{m}}\left[\left(\frac{h}{\rho_{1}}\right)^{2 m} \beta_{m}-\alpha_{m}\right] A_{m} .
\end{aligned}
$$

After multiplying both sides of this equation by $h^{m}$ and dividing by $m$, and then dividing each term of the resulting equation by (B.15), we obtain

$$
C L_{m}+\sum_{n=1}^{\infty}\left[\sigma_{m n}+(-1)^{n} T_{m n}\right] C_{n}=0, \quad m=1,2, \ldots,
$$

where

$$
\begin{gathered}
T_{m n}=\frac{h^{m}}{g_{m}-1} \frac{2}{\pi} \int_{0}^{\pi}\left[\left(\frac{n}{m}-g_{m}\right) \cos n \varphi^{\prime}-r \frac{n}{m} \frac{\cos (n+1) \varphi^{\prime}}{\sqrt{a+b \cos \varphi}}\right] \frac{\cos m \varphi}{(\sqrt{a+b \cos \varphi})^{n}} \mathrm{~d} \varphi, \\
L_{m}=\frac{h^{m}}{g_{m}-1}\left[\frac{r}{m} \frac{2}{\pi} \int_{0}^{\pi} \frac{(r+h \cos \varphi) \cos m \varphi}{a+b \cos \varphi} \mathrm{d} \varphi-g_{m} \frac{1}{\pi} \int_{0}^{\pi} \cos m \varphi \ln (a+b \cos \varphi) \mathrm{d} \varphi\right] .
\end{gathered}
$$

The term in the integrand of (B.18a), which includes the factor $n / m$, is transformed using (B.10a), and the resulting function

$$
\frac{\cos m \varphi \sin n \varphi^{\prime} \sin \varphi}{(\sqrt{a+b \cos \varphi})^{n+2}}
$$


is integrated by parts. Using (B.10b), we obtain

$$
\frac{n}{m} \frac{2}{\pi} \int_{0}^{\pi}\left[\cos n \varphi^{\prime}-r \frac{\cos (n+1) \varphi^{\prime}}{\sqrt{a+b \cos \varphi}}\right] \frac{\cos m \varphi}{(\sqrt{a+b \cos \varphi})^{n}} \mathrm{~d} \varphi=\frac{2}{\pi} \int_{0}^{\pi} \frac{\sin n \varphi^{\prime} \sin m \varphi}{(\sqrt{a+b \cos \varphi})^{n}} \mathrm{~d} \varphi .
$$

Thus, we obtain equation (21) instead of the expression for $T_{m n}$ (B.18a).

After integrating the second integral of (B.18b) by parts and rearranging, we obtain

$$
\frac{1}{\pi} \int_{0}^{\pi} \cos m \varphi \ln (a+b \cos \varphi) \mathrm{d} \varphi=\frac{2 r h}{\pi m} \int_{0}^{\pi} \frac{\sin m \varphi \sin \varphi}{a+b \cos \varphi} \mathrm{d} \varphi=\frac{2 r h}{\pi m} \int_{0}^{\pi} \frac{\cos (m-1) \varphi-\cos m \varphi \cos \varphi}{a+b \cos \varphi} \mathrm{d} \varphi,
$$

and this leads to

$$
L_{m}=\frac{h^{m}}{g_{m}-1} \frac{r}{m} \frac{2}{\pi} \int_{0}^{\pi}\left[\left(r-\frac{a\left(1+g_{m}\right)}{2 r}\right) \frac{\cos m \varphi}{a+b \cos \varphi}-h g_{m} \frac{\cos (m-1) \varphi}{a+b \cos \varphi}\right] \mathrm{d} \varphi .
$$

By applying formula 3.613.1 given in [10], we obtain

$$
\frac{1}{\pi} \int_{0}^{\pi} \frac{\cos n \varphi}{a+b \cos \varphi} \mathrm{d} \varphi=\frac{1}{r^{2}-h^{2}}\left(-\frac{h}{r}\right)^{n}
$$

It follows that

$$
L_{m}=\frac{(-1)^{m}}{m}\left(\frac{h^{2}}{r}\right)^{m} \frac{g_{m}+1}{g_{m}-1},
$$

which leads to the second equation in the system of equations (20).

We shall now derive expressions for the electric field components. In order to find $E_{x}$ and $E_{y}$, the quantities $\rho$, $\rho^{\prime}, \varphi, \varphi^{\prime}$ will be expressed as functions of coordinates $x, y$, measured from the origin $O$ :

$$
\rho=\sqrt{x^{2}+y^{2}}, \quad \rho^{\prime}=\sqrt{(x+r)^{2}+y^{2}}, \quad \varphi=\arccos \frac{x}{\sqrt{x^{2}+y^{2}}}, \quad \varphi^{\prime}=\arccos \frac{x+r}{\sqrt{(x+r)^{2}+y^{2}}} .
$$

We then obtain

$$
\begin{aligned}
\frac{\partial}{\partial x} \frac{\cos n \varphi}{\rho^{n}} & =-n \frac{\cos (n+1) \varphi}{\left(\sqrt{x^{2}+y^{2}}\right)^{n+1}}, & \frac{\partial}{\partial x} \frac{\cos n \varphi^{\prime}}{\rho^{\prime n}} & =-n \frac{\cos (n+1) \varphi^{\prime}}{\left(\sqrt{(x+r)^{2}+y^{2}}\right)^{n+1}}, \\
\frac{\partial}{\partial y} \frac{\cos n \varphi}{\rho^{n}} & =-n \frac{\sin (n+1) \varphi}{\left(\sqrt{x^{2}+y^{2}}\right)^{n+1}}, & \frac{\partial}{\partial y} \frac{\cos n \varphi^{\prime}}{\rho^{\prime n}} & =-n \frac{\sin (n+1) \varphi^{\prime}}{\left(\sqrt{(x+r)^{2}+y^{2}}\right)^{n+1}}
\end{aligned}
$$

where $\varphi$ and $\varphi^{\prime}$ are expressed according to (B.20). Using those equations in conjunction with equation (16), we obtain expressions for the vertical and horizontal components of electric field $E_{x}$ and $E_{y}$ in the air between toner and paper:

$$
\begin{aligned}
& E_{x}(x, y)=\left[\frac{x+r}{(x+r)^{2}+y^{2}}-\frac{x}{x^{2}+y^{2}}\right] C+\sum_{n=1}^{N} n C_{n}\left[\frac{\cos (n+1) \varphi}{\left(\sqrt{x^{2}+y^{2}}\right)^{n+1}}-(-1)^{n} \frac{\cos (n+1) \varphi^{\prime}}{\left(\sqrt{(x+r)^{2}+y^{2}}\right)^{n+1}}\right], \\
& E_{y}(x, y)=\left[\frac{y}{(x+r)^{2}+y^{2}}-\frac{y}{x^{2}+y^{2}}\right] C+\sum_{n=1}^{N} n C_{n}\left[\frac{\sin (n+1) \varphi}{\left(\sqrt{x^{2}+y^{2}}\right)^{n+1}}-(-1)^{n} \frac{\sin (n+1) \varphi^{\prime}}{\left(\sqrt{(x+r)^{2}+y^{2}}\right)^{n+1}}\right] .
\end{aligned}
$$


The radial and tangential components $E_{\rho}$ and $E_{\varphi}$ are calculated as functions of the angle $\varphi$ on the outer surface of the toner only, i. e., when $\rho=h$. Here, we use equalities (18) and formulas (B.4), (B.10b). Thus, we obtain

$$
\frac{\partial}{\partial \varphi} \frac{\cos n \varphi^{\prime}}{\rho^{\prime n}}=n h \frac{r \sin \varphi \cos n \varphi^{\prime}-(h+r \cos \varphi) \sin n \varphi^{\prime}}{(\sqrt{a+b \cos \varphi})^{n+2}} .
$$

We then have

$$
\begin{aligned}
& E_{\rho}=\frac{1}{h}\left\{-r \frac{r+h \cos \varphi}{a+b \cos \varphi} C+\sum_{n=1}^{N} n C_{n}\left[\frac{\cos n \varphi}{h^{n}}-(-1)^{n} \frac{\cos n \varphi^{\prime}-r \frac{\cos (n+1) \varphi^{\prime}}{\sqrt{a+b \cos \varphi}}}{(\sqrt{a+b \cos \varphi})^{n}}\right]\right\} \\
& E_{\varphi}=-\frac{r \sin \varphi}{a+b \cos \varphi} C+\sum_{n=1}^{N} n C_{n}\left[\frac{\sin n \varphi}{h^{n+1}}+(-1)^{n} \frac{r \sin \varphi \cos n \varphi^{\prime}-(h+r \cos \varphi) \sin n \varphi^{\prime}}{(\sqrt{a+b \cos \varphi})^{n+2}}\right],
\end{aligned}
$$

where

$$
\varphi^{\prime}=\arccos \frac{r+h \cos \varphi}{\sqrt{(a+b \cos \varphi)}}
$$

\section{References}

[1] M. Schleusener, in: 7th International Congress on Advances in Non-Impact Printing Technologies, Final Program and Proceedings, 167 (1991).

[2] M.C. Zaretsky, J. Imaging Sci. Technol. 37, 187 (1993).

[3] J.W. May and T.N. Tombs, in: IS\&T's NIP 13: International Conference on Digital Printing Technologies, 71 (1997).

[4] T.N. Tombs, in: IS\&T's NIP 14: International Conference on Digital Printing Technologies, 440 (1998).

[5] I. Chen and M.-K. Tse, in: IS\&T's NIP 15: International Conference on Digital Printing Technologies, 155 (1999).

[6] N. Provatas, A. Cassidy, and M. Inoue, in: IS\&T's NIP 20: International Conference on Digital Printing Technologies, 958 (2004).
[7] Y. Furuya, J. Imaging Sci. Technol. 45, 542 (2001).

[8] J. Kallunki, M. Alava, and E.K.O. Hellen, J. Appl. Phys. 100, 023528 (2006).

[9] T. Ito and H. Kawamoto, Int. J. Appl. Electromagnetics Mechanics 13, 85 (2001 / 2002).

[10] A. Cassidy, M. Grant, and N. Provatas, Modelling Simul. Mater. Sci. Eng. 12, 91 (2004).

[11] Ch. Tong, T. Wu, and N. Provatas, Modelling Simul. Mater. Sci. Eng. 14, 1447 (2006).

[12] I. Chen and M.-K. Tse, in: IS\&T's NIP 21: International Conference on Digital Printing Technologies, 566 (2005).

[13] R.M. Schaffert, Electrophotography, enl. rev. ed. (John Willey, New York, 1975).

[14] I.S. Gradshteyn, I.M. Ryzhik, A. Jeffrey, and D. Zwillinger, Table of Integrals, Series, and Products, 7 th ed. (Academic Press, 2007).

\title{
ELEKTROSTATINIO LAUKO DVIMATĖJE TONERINIO VAIZDO PERKĖLIMO SRITYJE PRIKLAUSOMYBE் NUO POPIERIAUS SAVYBIU
}

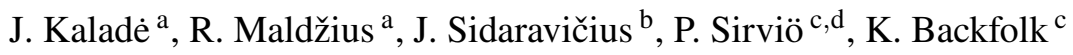 \\ ${ }^{\text {a }}$ Vilniaus universitetas, Vilnius, Lietuva \\ ${ }^{\mathrm{b}}$ Vilniaus Gedimino technikos universitetas, Vilnius, Lietuva \\ c „Stora Enso Oyj“ Imatros moksliniu tyrimu centras, Imatra, Suomija \\ d Åbo Akademi universitetas, Turku, Suomija
}

\section{Santrauka}

Tiksliai sprendžiant Puasono lygti, dvimačiu atveju apskaičiuotas elektrostatinio lauko stiprio pasiskirstymas lazerinio spausdintuvo tonerinio vaizdo perkèlimo srityje, popieriu apibūdinant dielektrinio storio artiniu. Ištirta elektrinio lauko stiprio vektoriaus sando, lygiagretaus popieriaus paviršiui, padètis ir jo vertès bei popieriaus paviršiui statmenas sandas ivvairiems popieriaus bei tonerio parametrų rinkiniams. Išanalizuotos Pašeno išlydžio tonerinio vaizdo perkèlimo srityje sąlygos. 